\title{
Exact Results on Some Nonlinear Laguerre-type Diffusion Equations
}

\section{Roberto Garra ${ }^{a}$ and Zivorad Tomovski ${ }^{b, c}$}

${ }^{a}$ Department of Statistics, Sapienza University of Rome, Italy Piazzale Aldo Moro 5, 00185 Roma, Italy

${ }^{b}$ Department of Mathematics, University of Ostrava, The Czech Republic

Dvořákova 7, 70103 Ostrava, Czech Republic

${ }^{c}$ Institute of Mathematics, Saints Cyril and Methodius University, North Macedonia

Arhimedova 3, 1000 Skopje, North Macedonia

E-mail(corresp.): roberto.garra@sbai.uniroma1.it

Received September 30, 2019; revised November 1, 2020; accepted November 3, 2020

\begin{abstract}
In this paper we obtain some new explicit results for nonlinear equations involving Laguerre derivatives in space and/or in time. In particular, by using the invariant subspace method, we have many interesting cases admitting exact solutions in terms of Laguerre functions. Nonlinear diffusive-type and telegraph-type equations are considered and also the space and time-fractional counterpart are analyzed, involving Caputo or Prabhakar-type derivatives. The main aim of this paper is to point out that it is possible to construct many new interesting examples of nonlinear diffusive equations with variable coefficients admitting exact solutions in terms of Laguerre and Mittag-Leffler functions.
\end{abstract}

Keywords: Laguerre derivatives, nonlinear partial differential equations, invariant subspace methods, exact solutions.

AMS Subject Classification: $37 \mathrm{~J} 15 ; 34 \mathrm{~A} 08 ; 35 \mathrm{R} 11$.

\section{Introduction and preliminaries}

In this note we apply the invariant subspace method [5] to solve nonlinear partial differential equations involving space and/or time Laguerre derivatives of first order

$$
D_{L}:=\frac{d}{d x} x \frac{d}{d x}
$$

Copyright (c) 2021 The Author(s). Published by Vilnius Gediminas Technical University This is an Open Access article distributed under the terms of the Creative Commons Attribution License (http://creativecommons.org/licenses/by/4.0/), which permits unrestricted use, distribution, and reproduction in any medium, provided the original author and source are credited. 
and $n$-order

$$
D_{n L}:=\frac{d}{d x} x \frac{d}{d x} x \ldots x \frac{d}{d x},
$$

that is the first order derivative applied to $n$-times the operator $x \frac{d}{d x}$.

It is well-known that a wide class of nonlinear partial differential equations admits a solution by separating variables in spaces generated by exponential, trigoniometric, hyperbolic and polynomial functions, according to the general theory developed by V. Galaktionov and S. Svirshchevskii in [5]. Here we consider some nonlinear equations with variable coefficients that admit solutions in terms of Laguerre (or Tricomi) functions and we will try to explain their possible meaning and utility in physics. Laguerre derivatives have been studied by different authors from the mathematical point of view (e.g. [4]) and for relevant applications. For example, in [1] population dynamics models have been studied by applying Laguerre derivatives. More recently, for example in [18], the applications of Laguerre derivatives in space have been studied in the context of heat propagation models. On the other hand, from the mathematical point of view, the role of Laguerre derivatives in the framework of the so called monomiality principle has been pointed out for example in [3]. Nonlinear equations involving Laguerre derivatives was not considered previously and the aim of this paper is to show that there are wide classes of nonlinear PDEs (also involging fractional derivatives) involving Laguerre derivatives that admit exact solutions. Many of these equations can be seen as a generalization of classical nonlinear models. This can be a starting point to understand the possible role of Laguerre derivatives in nonlinear models.

We first recall (see e.g. $[3,4]$ ) that the so-called Laguerre-exponential function or Tricomi function

$$
C_{0}(-x)=\sum_{k=0}^{\infty} \frac{(-x)^{k}}{(k !)^{2}}
$$

is an eigenfunction of the Laguerre derivative, i.e.

$$
\left(\frac{d}{d x} x \frac{d}{d x}\right) C_{0}(-\lambda x)=-\lambda C_{0}(-\lambda x) .
$$

Dattoli and Ricci in [4] considered $L$-circular and $L$-hyperbolic functions associated to the Tricomi function $C_{0}(-x)$. For example, the so-called $L$-circular functions are

$$
\cos _{1}(-x)=\sum_{k=0}^{\infty}(-1)^{k} \frac{x^{2 k}}{((2 k) !)^{2}}, \quad \sin _{1}(-x)=\sum_{k=0}^{\infty}(-1)^{k} \frac{x^{2 k+1}}{((2 k+1) !)^{2}} .
$$

Dattoli and Ricci in the same paper showed that the last trigonometric functions $\cos _{1}(-x)$ and $\sin _{1}(-x)$ are solutions of the classical harmonic equation of second order with Laguerre derivative:

$$
\left(D_{L}^{2}\right) u+u=0
$$

where $D_{L}^{2}=\frac{\partial^{2}}{\partial x^{2}} x^{2} \frac{\partial^{2}}{\partial x^{2}}$. 
Moreover, the function

$$
C_{1}(-x)=\sum_{k=0}^{\infty} \frac{(-x)^{k}}{(k !)^{3}}
$$

is an eigenfunction of the Laguerre derivative of second order, i.e.

$$
\left(\frac{d^{2}}{d x^{2}} x^{2} \frac{d^{2}}{d x^{2}}\right) C_{1}(-\lambda x)=-\lambda C_{1}(-\lambda x) .
$$

More generally it is possible to prove that

$$
C_{n}(-x)=\sum_{k=0}^{\infty} \frac{(-x)^{k}}{(k !)^{n+2}}
$$

solves the equation

$$
D_{n L} f(x)=-f(x) .
$$

In this paper we consider a number of exact results about nonlinear diffusivetype equations involving Laguerre derivatives. The solutions are obtained in a separating variable form by using the invariant subspace method or by generalized separating variable method. We also obtain some exact results for the space or time-fractional counterpart of these interesting equations involving Caputo or Prabhakar derivatives (see e.g. $[6,12]$ and the references therein).

\section{The invariant subspace method}

The invariant subspace method was introduced by Galaktionov and Svirschevskii in order to find exact explicit solutions for nonlinear partial differential equations, we refer in particular to [5] for a full treatment. Starting from a recent paper by Gazizov and Kasatkin [7], many papers have been devoted to the application of this method to find particular interesting classes of solutions also for fractional PDEs. We refer for example to the recent papers $[10,13,15,17]$. Indeed, there are few exact results regarding nonlinear fractional models that are motivated by different applications. The invariant subspace method is playing a central role to find new results for nonlinear physical models with memory or nonlocality that can be useful to better understand the main differences from the known classical models.

We here recall the main idea of this method. Let us consider for example a scalar evolution equation

$$
\frac{\partial u}{\partial t}=F\left[u, \frac{\partial u}{\partial x}, \ldots\right]
$$

where $u=u(x, t)$ and $F[\cdot]$ is a nonlinear differential operator. Given $n$ linearly independent functions $f_{1}(x), f_{2}(x), \ldots, f_{n}(x)$ we call $W_{n}$ the $n$-dimensional linear space $W_{n}=\left\langle f_{1}(x), \ldots, f_{n}(x)\right\rangle$. This space is called invariant under the given operator $F[u]$, if $F[y] \in W_{n}$ for any $y \in W_{n}$. This means that there exist $n$ functions $\Phi_{1}, \Phi_{2}, \ldots, \Phi_{n}$ such that

$$
F\left[C_{1} f_{1}(x)+\ldots C_{n} f_{n}(x)\right]=\Phi_{1}\left(C_{1}, \ldots, C_{n}\right) f_{1}(x)+\ldots+\Phi_{n}\left(C_{1}, \ldots, C_{n}\right) f_{n}(x),
$$


where $C_{1}, C_{2}, \ldots, C_{n}$ are arbitrary constants. Once the set of functions $f_{i}(x)$ that form the invariant subspace has been determined, we can search an exact solution of (2.1) in the invariant subspace in the form $u(x, t)=\sum_{i=1}^{n} u_{i}(t) f_{i}(x)$, where $f_{i}(x) \in W_{n}$. In this way, we arrive to a system of ODEs. In many cases, this problem is simpler than the original one and allows to find exact solutions by just separating variables [5]. We refer to the monograph [5] for further details and applications of this method.

It is not the aim of this paper to give a complete classification of the possible solutions that can be obtained by means of the invariant subspace method. Here we are going to study the solutions belonging to Laguerre exponential-invariant subspaces since we would like to understand the utility and applications of the known results regarding Laguerre derivatives in the context of nonlinear PDEs (also of fractional order). This choice is also motivated by the fact that these solutions are, in our view, the most interesting, in view of the central role played by $L$-functions to obtain meaningful mathematical results.

\section{$3 \quad$ Nonlinear Laguerre-type diffusion equations}

The following result plays a key-role in the construction of Laguerre-type invariant subspaces for nonlinear diffusion equations.

Theorem 1. The nonlinear Laguerre-type diffusion equation with quadratic absorption term

$$
\frac{\partial u}{\partial t}+u \frac{\partial}{\partial x} x \frac{\partial u}{\partial x}=-u^{2}
$$

admits a solution in the invariant subspace $W_{2}:\left\langle 1, C_{0}(-x)\right\rangle$ of the form

$$
u(x, t)=\frac{\left[1+C_{0}(-x)\right]}{\left(t_{0}+t\right)}, \quad t_{0}>0 .
$$

Proof. Equation (3.1) can be written as

$$
\frac{\partial u}{\partial t}=F\left[u, \frac{\partial u}{\partial x}, \frac{\partial^{2} u}{\partial x^{2}}\right]
$$

where

$$
F\left[u, \frac{\partial u}{\partial x}, \frac{\partial^{2} u}{\partial x^{2}}\right]:=-u \frac{\partial}{\partial x} x \frac{\partial u}{\partial x}-u^{2} .
$$

Since $F\left[c_{1}+c_{2} C_{0}(-x)\right]=-c_{1}^{2}-c_{1} c_{2} C_{0}(-x)$, the equation (3.1) admits the invariant subspace $W_{2}:\left\langle 1, C_{0}(-x)\right\rangle$ and therefore we can find a solution by separating variable of the form

$$
u(x, t)=a(t)+b(t) C_{0}(-x) .
$$

In order to find the explicit form of $a(t)$ and $b(t)$, substituting (3.2) in (3.1) we obtain the system of nonlinear coupled ODEs

$$
\dot{a}=-a^{2}, \quad \dot{b}=-a b,
$$


and we finally obtain the claimed result.

Regarding the physical meaning of the considered equation (3.1), we can observe that it coincides with a nonlinear diffusive equation involving an advective term, a quadratic absorption term and a nonlinear diffusive term, belonging to the more general class of equations

$$
\frac{\partial u}{\partial t}+u \frac{\partial u}{\partial x}+k(x, u) \frac{\partial^{2} u}{\partial x^{2}}=-u^{2}
$$

where $k(x, u)=x u$.

Remark 1. Since the function (1.3) solves the equation (1.4), by using similar methods we can treat also the higher order nonlinear diffusive equation

$$
\frac{\partial u}{\partial t}+u \partial_{L n, x} u=-u^{2}
$$

where $\partial_{L n, x}$ is the $n$-order Laguerre partial derivative (1.1) with respect to the $x$-variable. In this case we have that the higher order nonlinear equation (3.3) is solved by

$$
u(x, t)=\frac{\left[1+C_{n}(-x)\right]}{\left(t_{0}+t\right)}, \quad t_{0}>0 .
$$

In a similar way we can treat a wide family of nonlinear PDEs that admit solutions in the Laguerre-invariant subspace.

Remark 2. By using (1.2), it is possible to prove that the nonlinear equation

$$
\frac{\partial u}{\partial t}+u \frac{\partial^{2}}{\partial x^{2}} x^{2} \frac{\partial^{2}}{\partial x^{2}} u=-u^{2}
$$

admits a solution, given by

$$
u(x, t)=\frac{\left[c_{1} \cos _{1}(x)+c_{2} \sin _{1}(x)\right]}{\left(t_{0}+t\right)}, \quad t_{0}>0,
$$

where $c_{1}$ and $c_{2}$ are arbitrary constants.

\subsection{The time-fractional case}

In the recent literature, many studies have been devoted to the analysis of nonlinear space or time-fractional nonlinear equations by means of the invariant subspace method and the Lie group method (see for example $[2,7,11,14]$ ).

Here we consider nonlinear fractional equations involving Laguerre derivatives. This is a new interesting topic, since Laguerre derivatives introduce space or time-dependent coefficients in the nonlinear fractional equations that are generally not trivially solvable. We will use fractional derivatives in the sense of Caputo and in the sense of Prabhakar. This choice is essentially motivated by the central role played by these approaches in the theory of fractional calculus and its applications (see e.g. [12] and [16]). Obviously some of these results can be generalized in a simple way to other models involving Hadamard derivatives 
or tempered derivatives.

We first recall that the Caputo fractional derivative of order $\nu \in(0,1)$ is defined as

$$
D_{t}^{\nu} f(t)=\frac{1}{\Gamma(1-\nu)} \int_{0}^{t}(t-\tau)^{-\nu} \frac{d f}{d \tau} d \tau .
$$

Moreover, in the fractional calculus theory, a key role is played by the oneparameter Mittag-Leffler function

$$
E_{\nu}(\lambda t)=\sum_{k=0}^{\infty} \frac{(\lambda t)^{k}}{\Gamma(\nu k+1)}
$$

since it is an eigenfunction of the Caputo derivative, i.e.

$$
D_{t}^{\nu} E_{\nu}\left(-\lambda t^{\nu}\right)=-\lambda E_{\nu}\left(\lambda t^{\nu}\right)
$$

Recently, also the regularized Prabhakar derivative (see [6] and [16] for a full discussion) has gained a relevant interest in the literature in view of its physical applications. It is defined as

$$
{ }^{C} \mathcal{D}_{\rho, \mu, \lambda ; 0}^{\gamma} f(t)=\mathcal{E}_{\rho, m-\mu, \lambda ; 0}^{-\gamma}\left(\frac{d^{m}}{d t^{m}} f\right)(t),
$$

where $m=\lceil\mu\rceil$,

$$
\mathcal{E}_{\rho, \mu, \lambda ; a}^{\gamma} f(t)=\int_{0}^{t}(t-y)^{\mu-1} E_{\rho, \mu}^{\gamma}\left[\lambda(t-y)^{\rho}\right] f(y) d y
$$

is the Prabhakar fractional integral and

$$
E_{\rho, \mu}^{\gamma}(t)=\frac{1}{\Gamma(\gamma)} \sum_{k=0}^{\infty} \frac{\Gamma(\gamma+k) t^{k}}{k ! \Gamma(\rho k+\mu)}
$$

is the three-parameter Mittag-Leffler function. We refer to the monograph [9] for a complete reference about Mittag-Leffler function. Observe that the Prabhakar derivative (3.4) is a generalization of the Caputo derivative that is included as a special case for $\gamma=0$. Hereafter, we will consider in the following Theorems Prabhakar derivatives with $\gamma, \rho>0$ and $\mu \in(0,1)$. The more general case can be simply derived by using the results reported in Section 5 of the recent review paper on this topic [8].

It is possible to prove (by Laplace transform method) that a solution to the equation

$$
{ }^{C} \mathcal{D}_{\rho, \mu,-\lambda ; 0^{+}}^{\gamma} f(t)=-f(t)
$$

is given by

$$
f(t)=\sum_{k=0}^{\infty}\left(-t^{\mu}\right)^{k} E_{\rho, \mu k+1}^{\gamma k}\left(-\lambda t^{\rho}\right) .
$$

In analogy with the previous section, we can state the following 
Theorem 2. The nonlinear Laguerre-type diffusion equation with quadratic sink term

$$
\frac{\partial^{\nu}}{\partial t^{\nu}} u+u \frac{\partial}{\partial x} x \frac{\partial u}{\partial x}=-u^{2}
$$

admits a solution in the invariant subspace $W_{2}:\left\langle 1, C_{0}(-x)\right\rangle$ of the form

$$
u(x, t)=-\frac{\Gamma(1-\nu)}{\Gamma(1-2 \nu)} \frac{\left[1+C_{0}(-x)\right]}{t^{\nu}}, \quad t>0, \nu \in(0,1) \backslash\{1 / 2\} .
$$

Observe that we exclude the value $\nu=1 / 2$ due to the divergence of the Gamma function appearing in the solution. Moreover, the sign of the solution depends by $\nu$ because to the Gamma coefficients appearing in (3.6).

Theorem 3. The fractional nonlinear PDE with regularized Prabhakar time derivative

$$
{ }^{C} \mathcal{D}_{\rho, \mu,-\phi ; t}^{\gamma} u(x, t)+u \partial_{L n, x} u=-u^{2}-u
$$

admits a solution of the form

$$
u(x, t)=C_{n}(-x) \cdot \sum_{k=0}^{\infty}\left(-t^{\mu}\right)^{k} E_{\rho, \mu k+1}^{\gamma k}\left(-\lambda t^{\rho}\right) .
$$

In (3.7), we denoted by ${ }^{C} \mathcal{D}_{\rho, \mu,-\phi ; t}^{\gamma}$ the Prabhakar fractional partial derivative w.r.t. the t-variable.

Proof. By (1.3) and (1.4) we can find a solution in separating variable form $u(x, t)=C_{n}(-x) f(t)$ and by substituting $f(t)$ we have to solve equation (3.5), whose solution is given by (see [6])

$$
f(t)=\sum_{k=0}^{\infty}\left(-t^{\mu}\right)^{k} E_{\rho, \mu k+1}^{\gamma k}\left(-\lambda t^{\rho}\right) .
$$

Theorem 4. The fractional PDE with Prabhakar time derivative and Caputo space derivative of order $\beta \in(0,1)$ for $x \geq 0$

$$
{ }^{C} \mathcal{D}_{\rho, \mu,-\lambda ; t}^{\gamma} u(x, t)+u \frac{\partial^{\beta}}{\partial x^{\beta}} u(x, t)=-u^{2}-u
$$

admits a solution of the form

$$
u(x, t)=E_{\beta}\left(-x^{\beta}\right) \cdot \sum_{k=0}^{\infty}\left(-t^{\mu}\right)^{k} E_{\rho, \mu k+1}^{\gamma k}\left(-\lambda t^{\rho}\right) .
$$

Proof. Since the function

$$
E_{\beta}\left(-x^{\beta}\right)=\sum_{k=0}^{\infty} \frac{\left(-x^{\beta}\right)^{k}}{\Gamma(\beta k+1)},
$$

solves the equation

$$
\frac{\partial^{\beta}}{\partial x^{\beta}} h(x)=-h(x),
$$

we obtain the claimed result by direct calculations. 


\section{Nonlinear diffusive equations involving Laguerre time- derivatives}

We now consider the following nonlinear diffusive-type equation involving Laguerre time-derivatives

$$
\frac{\partial}{\partial t} t \frac{\partial}{\partial t} u=\frac{\partial}{\partial x} u^{\gamma} \frac{\partial u}{\partial x}-\lambda u, \quad \gamma>0
$$

The appearence of Laguerre derivatives in time lead to a telegraph-type equation with a non-linear diffusive term, suggesting the physical meaning of such equation. We have the following particular result

Theorem 5. The nonlinear diffusive-type equation (4.1) admits a solution of the form

$$
u(x, t)=C_{0}(-\lambda t) \cdot(x+c)^{\frac{1}{1+\gamma}},
$$

where $c$ is a real constant.

Proof. We first observe that the function $g(x)=\left[(x+c)^{\frac{1}{1+\gamma}}\right]$ is such that

$$
\frac{\partial}{\partial x} g^{\gamma} \frac{\partial g}{\partial x}=0
$$

Therefore we can find a solution in separating variable form $u(x, t)=g(x) f(t)$ and by substitution $f(t)$ must solve

$$
\frac{\partial}{\partial t} t \frac{\partial}{\partial t} f(t)=-\lambda f(t)
$$

as claimed.

Example 1. The nonlinear PDE

$$
u \frac{\partial^{2}}{\partial t^{2}} t^{2} \frac{\partial^{2}}{\partial t^{2}} u=\frac{\partial}{\partial x} u^{\gamma} \frac{\partial u}{\partial x}-u^{2}, \quad \gamma>0
$$

has a solution of the form

$$
u(x, t)=\left(c_{1} \cos _{1} t+c_{2} \sin _{1} t\right) \cdot(x+c)^{\frac{1}{1+\gamma}},
$$

where $c_{1}$ and $c_{2}$ are arbitrary constants.

Example 2. A nonlinear PDE

$$
\frac{\partial^{2}}{\partial t^{2}} t^{2} \frac{\partial^{2}}{\partial t^{2}} u=u \frac{\partial^{2}}{\partial x^{2}} x^{2} \frac{\partial^{2}}{\partial x^{2}} u-u+u^{2}
$$

has a solution of the form

$$
u(x, t)=\left(c_{1} \cos _{1} t+c_{2} \sin _{1} t\right)\left(c_{3} \cos _{1} x+c_{4} \sin _{1} x\right),
$$

where $c_{1}, c_{2}, c_{3}$ and $c_{4}$ are arbitrary constants. 
Example 3. The nonlinear Laguerre type PDE

$$
\frac{\partial}{\partial x} x \frac{\partial}{\partial x} u=u \frac{\partial}{\partial t} t \frac{\partial}{\partial t} u-\lambda u+\nu u^{2}
$$

admits a solution of the form

$$
u(x, t)=C_{0}(-\lambda x) \cdot C_{0}(-\nu t)
$$

We finally consider a multidimensional extension of the previous results about nonlinear diffusive equations involving Laguerre time-derivatives by employing the previous results, we have the following result

Theorem 6. The equation

$$
\frac{\partial}{\partial t} t \frac{\partial}{\partial t} u=\sum_{i=1}^{n} \frac{\partial}{\partial x_{i}} u^{\gamma_{i}} \frac{\partial u}{\partial x_{i}}-\lambda u, \quad \lambda \in R, \gamma_{i}>0
$$

admits a solution of the form

$$
u\left(x_{1}, x_{2}, \ldots, x_{n}, t\right)=C_{0}(-\lambda t) \cdot \prod_{i=1}^{n}\left(x_{i}+c_{i}\right)^{\frac{1}{1+\gamma_{i}}},
$$

where $c_{i}, i=1,2, \ldots, n$ are arbitrary real constants.

\section{Acknowledgements}

The work of R.G. has been carried out in the framework of the activities of GNFM.

Z.T. was supported by NWO [grant number 040.11.717 ], Department of Applied Mathematics, TU Delft.

\section{References}

[1] G. Bretti and P.E. Ricci. Laguerre-type special functions and population dynamics. Applied mathematics and computation, 187(1):89-100, 2007. https://doi.org/10.1016/j.amc.2006.08.106.

[2] S. Choudhary and V. Daftardar-Gejji. Invariant subspace method: a tool for solving fractional partial differential equations. Fractional Calculus and Applied Analysis, 20(2):477-493, 2017. https://doi.org/10.1515/fca-2017-0024.

[3] G. Dattoli, M.X. He and P.E. Ricci. Eigenfunctions of Laguerre-type operators and generalized evolution problems. Math. Comput. Model., 42:1263-1268, 2005. https://doi.org/10.1016/j.mcm.2005.01.034.

[4] G. Dattoli and P.E. Ricci. Laguerre-type exponents and the relevant L-circular and L-hyperbolic functions. Georgian Math. J., 20(2):477-493, 2017. 
[5] V. Galaktionov and S. Svirshchevskii. Exact solutions and invariant subspaces of nonlinear partial differential equations in mechanics and physics. Chapman and Hall/CRC applied mathematics and nonlinear science series, 2007. https://doi.org/10.1201/9781420011623.

[6] R. Garra, R.Gorenflo, F. Polito and Z. Tomovski. Hilfer-Prabhakar derivatives and some applications. Applied Mathematics and Computation, 242:576-589, 2014. https://doi.org/10.1016/j.amc.2014.05.129.

[7] R.K. Gazizov and A.A. Kasatkin. Construction of exact solutions for fractional order differential equations by the invariant subspace method. Computers \& Mathematics with Applications, 66(5):576-584, 2013. https://doi.org/10.1016/j.camwa.2013.05.006.

[8] A. Giusti, I. Colombaro, R. Garra, R. Garrappa, F. Polito, M. Popolizio and F. Mainardi. A practical guide to Prabhakar fractional calculus. Fractional Calculus and Applied Analysis, 23(1):9-54, 2020. https://doi.org/10.1515/fca2020-0002.

[9] R. Gorenflo, A.A. Kilbas, F. Mainardi and S.V. Rogosin. MittagLeffler functions, related topics and applications. Springer, Berlin, 2014. https://doi.org/10.1007/978-3-662-43930-2.

[10] M.S. Hashemi. Invariant subspaces admitted by fractional differential equations with conformable derivatives. Chaos, Solitons \& Fractals, 107:161-169, 2018. https://doi.org/10.1016/j.chaos.2018.01.002.

[11] A. Jannelli, M. Ruggieri and M.P. Speciale. Exact and numerical solutions of time-fractional advectiondiffusion equation with a nonlinear source term by means of the Lie symmetries. Nonlinear Dynamics, 92(2):543-555, 2018. https://doi.org/10.1007/s11071-018-4074-8.

[12] A.A. Kilbas, H.M. Srivastava and J.J. Trujillo. Theory and applications of fractional differential equations. Elsevier Science Limited, 2006.

[13] R. Najafi, F. Bahrami and M.S. Hashemi. Classical and nonclassical lie symmetry analysis to a class of nonlinear time-fractional differential equations. Nonlinear Dynamics, 87(3):1785-1796, 2017. https://doi.org/10.1007/s11071-016-3152-z.

[14] P. Prakash. New exact solutions of generalized convection-reactiondiffusion equation. European Physical Journal Plus, 134(6):261, 2019. https://doi.org/10.1140/epjp/i2019-12657-3.

[15] W. Rui. Idea of invariant subspace combined with elementary integral method for investigating exact solutions of time-fractional NPDEs. Applied Mathematics and Computation, 339(3):158-171, 2018. https://doi.org/10.1016/j.amc.2018.07.033.

[16] T. Sandev and Z. Tomovski. Fractional Equations and Models. Theory and Applications. Springer, 2019. https://doi.org/10.1007/978-3-030-29614-8.

[17] G.W. Wang and M.S. Hashemi. Lie symmetry analysis and soliton solutions of time-fractional $k(m, n)$ equation. Pramana, 88(1):7, 2017. https://doi.org/10.1007/s12043-016-1320-9.

[18] K.V. Zhukovsky. Operational solution for some types of second order differential equations and for relevant physical problems. Journal of Mathematical Analysis and Applications, 446(1):628-647, 2017. https://doi.org/10.1016/j.jmaa.2016.08.054. 